\title{
ESTIMATIVA DE PESO AO NASCIMENTO UTILIZANDO A ULTRASSONOGRAFIA BIDIMENSIONAL E TRIDIMENSIONAL
}

Luciano Marcondes Machado Nardozza ${ }^{*}$, Edward Araújo Junior², Marcio Fragoso Vieira ${ }^{3}$, Liliam Cristine Rolo4 ${ }^{4}$, Antonio Fernandes Moron ${ }^{5}$

Trabalho realizado no departamento de Obstetrícia da Universidade Federal de São Paulo - Escola Paulista de Medicina - UNIFESP-EPM, São Paulo, SP

*Correspondência:

Av. Lopes de Azevedo, 888

Morumbi

São Paulo - SP

CEP: 05603-001

Telefone: (11) 3815-1390

lunardozza@uol.com.br

\section{RESUMO}

OвJetivo. Avaliar a acurácia de uma fórmula utilizando o volume do braço (VolBra), da coxa (VolCox) e parâmetros bidimensionais [circunferência abdominal (CA) e comprimento do fêmur (CF)] fetais na predição de peso ao nascimento.

MÉTodos. Realizou-se um estudo do tipo corte transversal envolvendo 78 gestações únicas de fetos vivos, euploides e sem malformações estruturais, dentro de 48 horas antes do nascimento. 0 VolBra e o VolCox foram aferidos por meio da ultrassonografia tridimensional utilizando-se o modo multiplanar a intervalos de $5 \mathrm{~mm}$. A CA e o CF foram aferidos por meio da ultrassonografia bidimensional. Foram realizadas análises de regressão linear e polinomial para determinação da melhor fórmula para predizer o peso fetal com base no VolBra, VolCox, CA e CF. Os erros obtidos com essas fórmulas foram comparados com os obtidos com as fórmulas de Shepard e Hadlock por meio de ANOVA.

Resultados. A melhor fórmula que estimou o peso ao nascimento foi uma linear simples (Peso= $-1486,1+60,5 \mathrm{CA}+140,57 \mathrm{CF}+16,6 \mathrm{VolBra}+4,8 \mathrm{VolCox})$, com R2 = 0,932. O erro (E), erro absoluto (EA), erro percentual (EP) e erro percentual absoluto (EPA) da fórmula que utiliza os parâmetros bi e tridimensionais foram $0 \mathrm{~g}, 0,2 \%, 112,2 \mathrm{~g}$ e 3,7\%, respectivamente. Em relação às fórmulas bidimensionais, a nova equação apresentou menores E, EA, EP e EPA que a fórmula de Shepard, e menores EA e EPA em relação à fórmula de Hadlock.

Conclusão. A fórmula de predição de peso utilizando o VolBra, VolCox, CF e CA se mostrou superior às fórmulas que utilizam apenas parâmetros bidimensionais.

UNITERMOS: Imagem tridimensional. Peso fetal. Ultrassonografia.

\section{INTRODUÇÃO}

Os distúrbios do crescimento fetal são um capítulo importante dentre as patologias materno-fetais. Tanto a macrossomia ${ }^{1,2}$ quanto a restrição de crescimento fetal (RCF) $)^{3,4}$ estão relacionadas a um risco aumentado de morbidade e mortalidade perinatais, inclusive com consequências pós-natais tardias.

A estimativa do peso fetal é de fundamental importância no acompanhamento de situações obstétricas associadas com um crescimento fetal anormal ${ }^{5}$. É essencial que essa estimativa seja a mais precisa possível, para que possam ser tomadas decisões mais acertadas ${ }^{6}$. Por esses motivos, a estimativa do peso fetal é uma das mais importantes tarefas no acompanhamento pré-natal.
Tentativas iniciais para estimar o peso fetal por meio da ultrassonografia bidimensional (US2D) eram feitas utilizandose medidas fetais individualmente como o diâmetro biparietal $(D B P)^{7}$ ou a circunferência abdominal $(C A)^{8}$. Estudos subsequentes demonstraram que a utilização de múltiplas medidas fetais melhorava a acurácia da estimativa do peso fetal. Em nosso meio, as fórmulas mais utilizadas para predição de peso fetal são as de Shepard et al ${ }^{9}$. e Hadlock et al. ${ }^{10}$ Apesar do uso difundido da US2D para estimar o peso fetal, alguns modelos de predição comumente utilizados podem gerar erros de até 15\% do peso real ${ }^{10}$. Existe uma tendência a superestimar o peso em fetos menores e subestimar o peso em fetos macrossômicos. Na prática, o intervalo de confiança (IC) de $95 \%$ excede $14 \%$ do peso real em todas as fórmulas ${ }^{11}$.

1. Mestre, Doutor e Professor Adjunto do Departamento de Obstetrícia da Universidade Federal de São Paulo - UNIFESP, São Paulo, SP

2. Mestre e Doutor pelo Departamento de Obstetrícia da Universidade Federal de São Paulo - UNIFESP e Professor Afiliado do Departamento de Obstetrícia da Universidade Federal de São Paulo - UNIFESP, São Paulo, SP

3. Mestre pelo Departamento de Obstetrícia da Universidade Federal de São Paulo - UNIFESP e Pós-Graduando do Departamento de Obstetrícia da Universidade Federal de São Paulo - UNIFESP, São Paulo, SP

4. Mestre pelo Departamento de Obstetrícia da Universidade Federal de São Paulo - UNIFESP e Pós-Graduanda do Departamento de Obstetrícia da Universidade Federal de São Paulo - UNIFESP, São Paulo, SP

5. Professor Titular Livre-Docente do Departamento de Obstetrícia da Universidade Federal de São Paulo - UNIFESP e Professor Titular do Departamento de Obstetrícia da Universidade Federal de São Paulo UNIFESP, São Paulo, SP 
A utilização do volume de membros calculado pela US2D para avaliar o crescimento e estado nutricional do feto foi primeiramente sugerido em 1985. Concluiu-se que o volume de membros era altamente correlacionado com a idade gestacional e que poderia ser utilizado como possível preditor para RCF ${ }^{12}$. Nesse estudo, o volume do braço (VolBra) e o volume da coxa (VolCox) eram calculados com base no comprimento do membro e nos seus diâmetros transverso e ântero-posterior. Assumia-se, erroneamente, que o membro tivesse a forma de um cilindro.

Mais recentemente, a introdução da ultrassonografia tridimensional (US3D) tem desencadeado o ressurgimento da avaliação volumétrica dos membros fetais e, indiretamente, do crescimento e da nutrição fetal ${ }^{13}$. Como o volume de membros fetais, principalmente o VolBra e o VolCox, já estão bem estabelecidos como marcadores de crescimento e nutrição, diversos estudos têm utilizado estes parâmetros como preditores de peso ao nascimento, obtendo-se resultados mais fidedignos do que as fórmulas tradicionais utilizadas pela US2D ${ }^{14-16}$.

A maioria dos trabalhos de predição de peso por meio do volume de membros fetais obteve a sua fórmula avaliando populações etnicamente homogêneas (asiáticos e caucasianos), o que talvez dificulte a utilização dessas fórmulas em uma população predominantemente miscigenada. A realização de um estudo semelhante, porém com uma amostragem da população brasileira (etnicamente heterogênea), certamente seria mais adequada e conseguiria se aproximar mais da realidade brasileira.

O objetivo deste artigo é determinar uma fórmula para predição de peso fetal por meio da ultrassonografia tridimensional, com base no volume do braço e da coxa, associados a parâmetros bidimensionais e comparar o peso fetal estimado por essa fórmula com o peso estimado através das fórmulas de Hadlock e Shepard.

\section{Métodos}

Realizou-se um estudo tipo transversal, conduzido no Setor de Ultrassonografia Tridimensional do Departamento de Obstetrícia da UNIFESP, envolvendo um grupo de gestantes que tiveram seus partos realizados no Centro Obstétrico do Hospital São Paulo da Universidade Federal de São Paulo (UNIFESP). As pacientes selecionadas foram esclarecidas sobre os objetivos e a forma de realização do estudo e as que consentiram em participar voluntariamente assinaram o Termo de Consentimento Livre e Esclarecido (TCLE). A presente pesquisa foi aprovada pelo Comitê de Ética em Pesquisa da UNIFESP.

Somente foram incluídas gestações únicas de fetos vivos, que não apresentassem indícios de aneuploidia ou malformação estrutural, detectadas durante o período pré-natal. Foram considerados como critérios de exclusão: detecção de malformações estruturais após o nascimento ou suspeita de aneuploidias no exame neonatal; fetos natimortos; e ocorrência do parto em um período superior a 48 horas após a realização do exame ultrassonográfico. Todas as pacientes foram examinadas uma vez e por apenas um examinador.

Após a assinatura do TCLE, foi realizado o preenchimento do instrumento de coleta de dados, no qual constavam dados da paciente, da gestação, da ultrassonografia, do parto e do recémnascido (RN). Em seguida, as pacientes foram submetidas a um exame ultrassonográfico, descrito mais detalhadamente adiante.

Após o parto, os RN foram assistidos por neonatologistas que, após os cuidados iniciais, verificaram o índice de Apgar no primeiro e quinto minutos e realizaram a aferição do peso em balança eletrônica da marca Filizola ${ }^{\circledR}$ modelo BP Baby ${ }^{\circledR}$ (São Paulo, SP, Brasil) com divisões de 5 gramas.

Todos os exames foram realizados utilizando-se os aparelhos SONOACE 8000 Live e ACCUVIX XQ (Medison, Seoul, Korea). As medidas biométricas bidimensionais e a aquisição dos blocos de volumes tridimensionais eram realizadas utilizando-se um transdutor convexo volumétrico multifrequencial de varredura automática (3D4-7EK). O equipamento foi ajustado no momento de cada exame de modo a obter a melhor qualidade de imagem.

Inicialmente eram realizadas as medidas dos parâmetros bidimensionais (DBP, CC, CA, CF e CU) segundo a técnica padrão ${ }^{17-19}$. Também era feita a medida do índice de líquido amniótico (ILA) segundo a técnica dos quatro quadrantes ${ }^{20}$ e a avaliação do grau de maturação placentária ${ }^{21}$. A estimativa do peso fetal era feita com base nas fórmulas de Shepard et $\mathrm{al}^{9}$ e Hadlock et al. ${ }^{10}$

A seguir, passava-se para a avaliação tridimensional da coxa e do braço. Para o cálculo do VolCox, posicionava-se o transdutor no plano padrão de medida do fêmur. Quando o feto estava em repouso, acionava-se a tecla 3D no aparelho, abrindo assim a caixa de volume tridimensional e, quando todo o contorno do fêmur e da coxa eram visibilizados, pressionava-se a tecla "freeze" iniciando a varredura. Os aparelhos eram configurados previamente para realização da varredura com ângulo de $75^{\circ}$, com um tempo de varredura normal (aproximadamente quatro segundos). Desta forma, obtinha-se o modo multiplanar: plano sagital ou $A$, plano axial ou B e plano coronal ou C.

Selecionava-se a opção 2D, em seguida o plano A, girandose a coxa em torno do eixo "z", até que a diáfise femoral estivesse disposta horizontalmente. Posteriormente, deslocava-se o cursor até a extremidade proximal. Selecio- nava-se o plano B e deslocava-se o cursor ao longo do fêmur até a sua extremidade distal, em intervalos de 5,0 mm. Simultaneamente a cada deslocamento, a superfície externa da coxa no plano axial era delineada de forma manual, determinando-se uma área. Ao final do deslocamento, o programa calculava automaticamente o volume da coxa fetal (Figura 1). Para o cálculo do VolBra utilizouse técnica semelhante, sendo que a única diferença era o fato de se utilizar o plano padrão de medida do úmero.

Para calcular o número de gestantes necessário para realização deste estudo, utilizou-se a distância média aceitável (precisão) de 50 g com confiança de $95 \%$ e erro padrão de 194,3g supondo uma correlação média de cada medida volumétrica com o peso de 0,92 22 , dados estes baseados em estudo anterior ${ }^{15}$.

$(\mathrm{s} / \mathrm{r})^{2}$

$\mathrm{n}=$

[ precisão $\left./ \mathrm{Zg}_{/ 2}\right]^{2}$

onde:

$\mathrm{Zg}_{/ 2}$ é o valor da distribuição normal padrão de ordem g/2;

$\mathrm{s}^{2}$ é a variabilidade do peso ao nascimento;

$r$ é a correlação entre o peso e a medida de volume;

precisão é o máximo que se pretende se afastar da real média na população.

O tamanho amostral $(n)$, calculado com base na fórmula acima, foi de 80 pacientes. 
Figura 1 - Técnica proposta para medida do volume de membros fetais. Após a aquisição dos planos no modo multiplanar, selecionase o plano A e gira-se a coxa em torno do eixo " $z$ " de modo a dispor a diáfise horizontalmente. Em seguida, desloca-se 0 cursor até a extremidade proximal da diáfise. Neste momento, realiza-se a primeira delineação manual da superfície externa do membro no plano axial, determinando-se uma área.

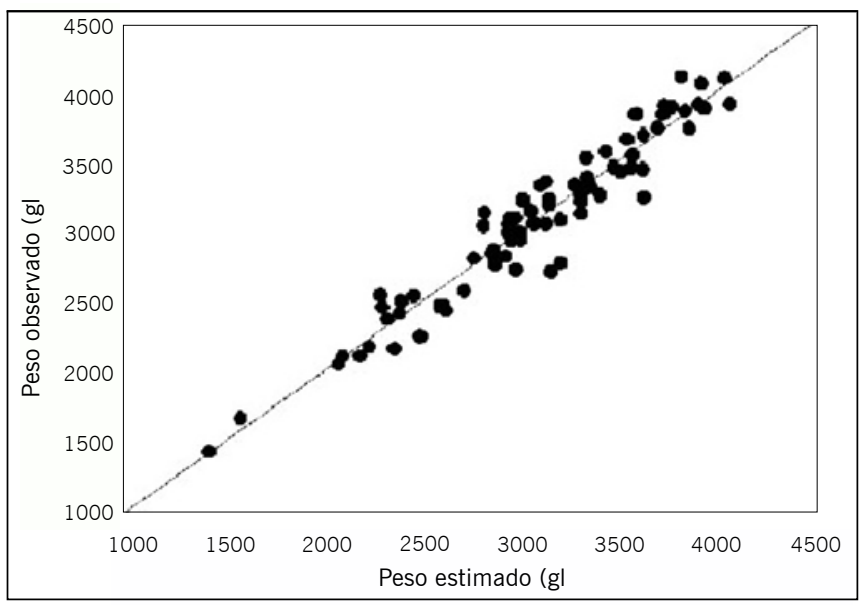

Todos os dados foram armazenados em planilha do programa Excel $2007^{\circledR}$ (Microsoft, Redmond, WA, USA) e analisados no programa Statistical Package for the Social Sciences (SPSS) versão 15.0 para Windows ${ }^{\circledR}$ (Chicago, IL, USA). Adotou-se o nível de significância de 5\% para todos os testes. Inicialmente, caracterizou-se a amostra estudada por meio da estatística descritiva, analisando-se os dados das pacientes, das gestações e dos RNs. Todas as variáveis foram expressas em média \pm desvio padrão (dp) (valores mínimo e máximo).

Os dados foram submetidos à análise de regressão linear e polinomial, para a obtenção das melhores fórmulas de predição do peso utilizando-se os parâmetros bidimensionais (DBP, CC, CA, CF) e tridimensionais (VolBra e VolCox). Comparouse o peso estimado por estas fórmulas com o peso estimado através da fórmula de Shepard et al. ${ }^{9}$ e das quatro fórmulas de Hadlock et al. ${ }^{10}$, tendo o peso ao nascimento como referência. Para cada fórmula, calculou-se o erro, o erro percentual, o erro absoluto e o erro percentual absoluto. Para comparação entre as fórmulas, utilizou-se análise de variâncias (ANOVA) com correção de Brown-Forsythe devido à falta de homocedasticidade (igualdade de variâncias) entre os grupos, testada pelo teste de Levene $(p<0,05)$. Posteriormente às ANOVAs, realizaram-se comparações múltiplas de Dunnett para variâncias desiguais para verificar quais dos modelos apresentavam menor erro diante dos demais ${ }^{23}$

\section{Resultados}

Oitenta e nove pacientes foram incluídas no estudo. Destas, oito foram excluídas porque o parto ocorreu em um período superior a 48 horas após a realização do exame. As características da população estudada estão demonstradas na Tabela 1.

Após realização de análise de regressão linear e polinomial
Tabela 1 - Características da amostra estudada (variáveis numéricas, $\mathrm{n}=81$ )

\begin{tabular}{ll}
\hline Variável & Média \pm dp (min - max) \\
\hline Idade da gestante (anos) & $27,4 \pm 6,54(15-42)$ \\
Idade gestacional* (semanas) & $38,5 \pm 1,86(32,3-41,4)$ \\
Peso ao nascimento (gramas) & $3105,8 \pm 553,90(1510-4065)$ \\
Intervalo** (horas) & $10,7 \pm 10,79(0,5-46,4)$ \\
Diâmetro biparietal (cm) & $8,8 \pm 0,47(7,7-9,7)$ \\
Circunferência cefálica (cm) & $32,4 \pm 1,58(28,2-35,5)$ \\
Circunferência abdominal (cm) & $33,2 \pm 2,52(25,1-38,0)$ \\
Comprimento do fêmur (cm) & $7,0 \pm 0,38(5,7-7,8)$ \\
Comprimento do úmero (cm) & $6,2 \pm 0,30(5,2-6,8)$ \\
Volume do braço (cm³) & $57,7 \pm 12,99(21,7$ - 82,6) \\
Volume da coxa (cm³) & $131,7 \pm 33,94(48,9$ - 207,3) \\
\hline dp: desvio padrão; min: valor mínimo; max: valor máximo; \\
* Idade gestacional no momento do exame; ** Intervalo entre o exame e o parto
\end{tabular}

até a quarta ordem, determinaram-se várias fórmulas para predição de peso utilizando parâmetros bidimensionais e tridimensionais. Dentre estas, a melhor e mais simples utilizava o VolBra, VolCox, CA e CF (Peso $=-1486,1$ 60,5CA 140,57CF 16,6VolBra 4,8VolCox).

Para avaliar a acurácia da fórmula, realizou-se uma comparação entre esta, a fórmula de Shepard et $\mathrm{al}^{9} \mathrm{e}$ as quatro fórmulas desenvolvidas por Hadlock et al. ${ }^{10}$ Utilizaram-se as medidas do erro, erro percentual, erro absoluto e erro percentual absoluto para tal comparação.

Verificou-se que a fórmula que associava parâmetros bi e tridimensionais apresentava menor erro $(0 \mathrm{~g})$ e erro percentual $(-0,2 \%)$ com menores desvios padrão que as demais, embora somente a diferença em relação à fórmula de Shepard et al. ${ }^{9}$ tenha sido estatisticamente significativa. 0 erro absoluto médio (112,2 g) e o erro percentual absoluto médio (3,7\%), assim como seus respectivos desvios padrão, foram menores que todas as demais fórmulas, diferenças estas consideradas significativas do ponto de vista estatístico (Tabela 2).

\section{Discussão}

Desde o início da utilização da ultrassonografia, diversos parâmetros biométricos fetais foram empregados, isoladamente ou em conjunto, na predição de peso ${ }^{7-10}$. Os parâmetros fetais mais utilizados são o DBP, CC, CA e CF, todos obtidos por meio da US2D. Outro parâmetro importante para a predição de peso é a medida da circunferência da coxa. Em estudo pioneiro, Favre et al. ${ }^{24}$ comprovaram que a fórmula que utilizava a circunferência da coxa fetal, obtida pela US3D, foi mais acurada na predição de peso de fetos pequenos para a idade gestacional que a fórmula que utilizava múltiplos parâmetros. Um grande prejuízo desse método é não considerar as partes moles (músculo e tecido 


\begin{tabular}{|c|c|c|c|c|c|c|c|c|}
\hline \multicolumn{9}{|c|}{$\begin{array}{c}\text { Tabela } 2 \text { - Comparação dos erros resultantes da fórmula que utiliza } \\
\text { parâmetros bi e tridimensionais, e das fórmulas de Hadlock e } \\
\text { Shepard, com resultado do teste estatístico }\end{array}$} \\
\hline & $E(g)$ & $p$ & ) & $p$ & & $p$ & $\begin{array}{l}\text { EPA } \\
(\%)\end{array}$ & $p$ \\
\hline 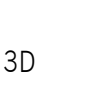 & $\begin{array}{l}0,0 \pm \\
146,2\end{array}$ & & $\begin{array}{c}-0,2 \pm \\
4,9\end{array}$ & & $\begin{array}{c}112,2 \pm \\
92,9\end{array}$ & & 3,2 & \\
\hline AUI & $\begin{array}{l}5,4 \pm \\
245,4\end{array}$ & 1,000 & $\begin{array}{c}0,2 \pm \\
7,8\end{array}$ & 1,000 & $\begin{array}{c}193,8 \pm \\
149,0\end{array}$ & 0,003 & 4,7 & 0,008 \\
\hline U & $\begin{array}{c}-58,9 \pm \\
236,6\end{array}$ & 0,953 & $\begin{array}{c}-1,5 \pm \\
7,4\end{array}$ & 1,000 & $\begin{array}{c}191,8 \pm \\
149,1\end{array}$ & 0,0 & $\begin{array}{c}6,1 \pm \\
4,5\end{array}$ & 0,009 \\
\hline AD3 & $\begin{array}{c}-72,2 \pm \\
243,6\end{array}$ & 0,715 & $\begin{array}{c}-1,9 \pm \\
7,5\end{array}$ & 0,991 & $\begin{array}{c}199,2 \pm \\
156,5\end{array}$ & 0,002 & $\begin{array}{c}6,3 \pm \\
4,4\end{array}$ & 0,003 \\
\hline HAD4 & $\begin{array}{c}-78,6 \pm \\
239,2\end{array}$ & 0,497 & $\begin{array}{c}-2,1 \pm \\
7,4\end{array}$ & 0,936 & $\begin{array}{c}196,8 \pm \\
155,9\end{array}$ & 0,003 & $\begin{array}{c}6,2 \pm \\
4,5\end{array}$ & 0,005 \\
\hline SHEP & $\begin{array}{c}-128,0 \\
\pm 268,7\end{array}$ & 015 & $\begin{array}{c}-3,9 \pm \\
8,4\end{array}$ & 0,045 & $\begin{array}{c}236,7 \pm \\
179,0\end{array}$ & 0,001 & $\begin{array}{c}7,5 \pm \\
5,4\end{array}$ & $<0,001$ \\
\hline
\end{tabular}

Valores expressos em média \pm desvio-padrão; $\mathrm{E}$ (erro): peso estimado-peso observado

EP (erro percentual): 100x(peso estimado- peso observado)/peso observado; EA (erro absoluto): $1 / 2$ peso estimado-peso observado $1 / 2$; EPA (erro percentual absoluto): $1 / 2100 \times$ (peso estimado- peso observado)/peso observado $1 / 2$

3D: Peso $=-1486,1+(60,5 x C A)+(140,6 x F)+(16,6 x$ VolBra $)+(4,8 x$ VolCox $)$

Hadlock 1: $\log _{10}($ Peso $)=1,304+(0,05281 \times C A)+(0,1938 \times F)-(0,004 \times C A x F)$

Hadlock 2: $\log _{10}($ Peso $)=1,335-(0,0034 x$ CAxF $)+(0,0316 x D B P)+(0,0457 x C A)+(0,1623 x F)$

Hadlock 3: $\log _{10}($ Peso $)=1,326-(0,00326 x C A x F)+(0,0107 x C C)+(0,0438 x C A)+(0,158 x F)$

Hadlock 4: $\log _{10}($ Peso $)=1,3596-(0,00386 \times C A x F)+(0,0064 \times C C)+(0,00061 \times D B P \times C A)+$ $(0,0424 \times C A)+(0,174 x F)$

Shepard: $\log _{10}($ Peso $)=-1,7492+(0,166 \times$ DBP $)+(0,046 \times C A)-0,002646(C A x D B P)$

p: resultado da ANOVA, nível de significância de $5 \%$

celular subcutâneo) na estimativa do peso fetal. Sabe-se que as partes moles, apesar de constituírem apenas $14 \%$ do peso de um RN, explicam $46 \%$ de sua variação ${ }^{25}$.

Há mais de duas décadas, já se demonstrava a importância das partes moles, analisada de forma indireta pelo volume de membros fetais, aferido por meio da US2D, como parâmetro de avaliação do crescimento e estado nutricional do feto ${ }^{18}$. Percebe-se que diversas fórmulas já utilizam o volume de membros calculado por meio da US3D (associados ou não a parâmetros bidimensionais) para predição de peso fetal ${ }^{14-}$ 16,26-28. Entretanto, nenhuma foi desenvolvida com base na população brasileira.

A amostragem deste estudo foi constituída por 81 gestantes, sendo que todos os exames foram realizados dentro de 48 horas antes do parto, à semelhança de outros estudos ${ }^{9,14-16,29}$. Outros pesquisadores optaram por intervalos que variavam de quatro $^{30}$ a sete dias ${ }^{10,17,26,27}$. O intervalo médio entre a realização do exame e o parto obtido neste estudo foi de 10,6 horas. Acredita-se que quanto menor esse intervalo, menor seria a variação de peso, o que possibilitaria a determinação de uma fórmula mais precisa.

A idade média das gestantes foi de 27,4 anos. Se comparada com todos os demais trabalhos já citados, é a média de idade mais baixa. A idade gestacional média (38,5 semanas) foi bem semelhante aos demais, com exceção de um, em que a idade gestacional média situava-se abaixo de 37 semanas $^{26}$. 0 peso médio ao nascimento foi de $3.105 \pm 553,9 \mathrm{~g}$, este se mostrou semelhante ao obtido por outros pesquisadores ${ }^{15}$ (3.070 \pm $586,7 \mathrm{~g})$.

Optou-se por realizar a medida do volume dos membros através do modo multiplanar, a intervalos de $5 \mathrm{~mm}$. Outros autores utilizaram intervalos de $3 \mathrm{~mm}^{14,15}$. Pang et al. ${ }^{31}$ compararam a acurácia da US3D em aferir volumes utilizando vários números de planos de imagens. Volumes mais acurados foram obtidos com o máximo número de cortes. Entretanto, a redução do número de cortes não levou a uma piora nos resultados e ainda teve a vantagem de diminuir em $50 \%$ o tempo necessário para a realização da medida.

Com a utilização da fórmula com parâmetros bi e tridimensionais, obteve-se um erro médio $(0,00 \pm 146,2 \mathrm{~g})$ menor que todas as outras fórmulas, embora somente em relação à fórmula de Shepard et al. ${ }^{9}(-128,03 \pm 268,67$ g) essa diferença foi significativa $(p=0,015)$. Percebeu-se ainda que todos os erros médios das fórmulas bidimensionais foram negativos, o que significa que elas subestimavam o peso fetal na amostra estudada. Também houve diferença estatisticamente significativa entre o erro percentual médio $(-0,2 \pm 4,9 \%)$ e da fórmula de Shepard et al. ${ }^{9}$ que foi de $-3,9 \pm 8,4 \%(p=0,045)$. Em relação ao erro absoluto médio $(112,2 \pm 92,9 \mathrm{~g})$ e ao erro percentual absoluto médio $(3,7$ $\pm 3,2 \%$ ), estes se mostraram menores que todas as fórmulas de Hadlock e de Shepard $(p<0,05)$. Quando se comparou os valores dos erros obtidos por este estudo com os obtidos por Schild et al. ${ }^{26}$ (erro absoluto médio 155,2 $\pm 118,2$ g) e Lee et al. ${ }^{30}$ (erro percentual médio $-0,025 \pm 7,8 \%$ ) verificou-se que são bastante semelhantes.

Constatou-se que a utilização do VolBra e do VolCox, associados ao CF e CA melhorou a acurácia da predição de peso em nossa amostra, quando comparadas às outras fórmulas que utilizam somente parâmetros bidimensionais, principalmente em relação à fórmula de Shepard et al ${ }^{9}$. Tal fato, já havia sido sugerido por Schild et al. ${ }^{26}$ que obtiveram melhor precisão na associação do VolBra, VolCox, volume abdominal e DBP. Entretanto, novos estudos prospectivos e com casuísticas maiores são necessários para confirmação destes resultados, principalmente em relação às fórmulas de Hadlock et al. ${ }^{10}$, que não apresentaram diferenças estatisticamente significativas em relação ao erro médio e ao erro percentual médio.

Pelo que se sabe, este é o primeiro estudo nacional que utiliza o volume de membros fetais aferidos pela US3D associado a parâmetros bidimensionais para predição de peso fetal. Apesar de este método necessitar de um espaço de tempo maior e de profissionais especializados para sua realização, é mais uma ferramenta que se pode contar no manejo de casos mais específicos.

\section{Conclusão}

A fórmula de predição de peso utilizando o VolBra, VolCox, $\mathrm{CF}$ e CA se mostrou superior às fórmulas que utilizam apenas parâmetros bidimensionais. 


\section{Suporte Financeiro:}

FAPESP nº 07/54402-1, Auxílio Pesquisa

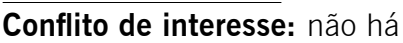

\section{SUMmary}

\section{ESTIMATE OF BIRTH WEIGHT USING TWO- AND THREE-DIMENSIONAL ULTRASONOGRAPHY}

OBJective. Assess and compare accuracy of birth weight prediction using a combination of two-dimensional (abdominal circumference - AC and femur length - FL) and threedimensional parameters (fetal arm -VolArm and thigh -VolTh volumes).

Methods. A cross sectional study was carried out involving 78 singleton, live, euploid fetuses without structural malformations born within 48 hours after ultrasonography. VolArm and VolTh were obtained by three-dimensional ultrasound using the multiplanar mode at $5 \mathrm{~mm}$ intervals. AC and FL were measured by two-dimensional ultrasound. Linear and polynomial regressions were calculated to determine the best formula to predict birth weight using VolArm, VolTh, CA and FL. ANOVA was used to compare errors in birth weight prediction using this formula and those obtained using the Shepard and Hadlock formulae.

RESULTS. The best formula for prediction of birth weight was a simple linear regression (Weight $=-1486.1+60.5 A C+$ 140.57FL + 16.6VolArm + 4.8VolTh), R2=0.932. The error (E), absolute error ( $A E)$, percent error (PE) and absolute percent error (APE) for this new formula were $0 \mathrm{~g}, 0.2 \%, 112.2 \mathrm{~g}$ and 3.7\%. This new formula had smaller $E, A E, P E$ and APE than the Shepard formula and smaller AE and APE than Hadlock's formula.

Conclusion. A formula using VolArm, VolTh, AC and FL was more accurate for prediction of birth weight than formulae using only two-dimensional parameters. [Rev Assoc Med Bras 2010; 56(2): 204-8]

KEY wORDS: Fetal weight. Imaging. Three-Dimensional. Ultrasonography.

\section{REFERÊNCIAS}

1. Boulet SL, Salihu HM, Alexander GR. Mode of delivery and birth outcomes of macrosomic infants. J Obstet Gynaecol. 2004;24(6):622-9.

2. Raio L, Ghezzi F, Di NE, Buttarelli M, Franchi M, Durig $P$, et al. Perinatal outcome of fetuses with a birth weight greater than $4500 \mathrm{~g}$ : an analysis of 3356 cases. Eur J Obstet Gynecol Reprod Biol. 2003;109(2):160-5.

3. Dashe JS, Mclntire DD, Lucas MJ, Leveno KJ. Effects of symmetric and asymmetric fetal growth on pregnancy outcomes. Obstet Gynecol. 2000;96(3):321-7

4. Roth S, Chang TC, Robson S, Spencer JA, Wyatt JS, Stewart AL. The neurodevelopmental outcome of term infants with different intrauterine growth characteristics. Early Hum Dev. 1999;55(1):39-50.

5. Favre R, Nisand G, Bettahar K, Grange G, Nisand I. Measurement of limb circumferences with three-dimensional ultrasound for fetal weight estimation. Ultrasound Obstet Gynecol. 1993;3(3):176-9.

6. Schild RL. Three-dimensional volumetry and fetal weight measurement. Ultrasound Obstet Gynecol. 2007;30(6):799-803.
7. Ianniruberto A, Gibbons JM Jr. Predicting fetal weight by ultrasonic B-scan cephalometry. An improved technic with disappointing results. Obstet Gynecol. 1971;37(5):689-94

8. Campbell S, Wilkin D. Ultrasonic measurement of fetal abdomen circumference in the estimation of fetal weight. Br J Obstet Gynaecol. 1975;82(9):689-97.

9. Shepard MJ, Richards VA, Berkowitz RL, Warsof SL, Hobbins JC. An evaluation of two equations for predicting fetal weight by ultrasound. Am J Obstet Gynecol. 1982;142(1):47-54.

10. Hadlock FP, Harrist RB, Sharman RS, Deter RL, Park SK. Estimation of fetal weight with the use of head, body, and femur measurements--a prospective study. Am J Obstet Gynecol. 1985;151(3):333-7.

11. Dudley NJ. A systematic review of the ultrasound estimation of fetal weight. Ultrasound Obstet Gynecol. 2005;25(1):80-9.

12. Jeanty P, Romero R, Hobbins JC. Fetal limb volume: a new parameter to assess fetal growth and nutrition. J Ultrasound Med. 1985;4(6):273-82.

13. Zelop CM. Prediction of fetal weight with the use of three-dimensional ultrasonography. Clin Obstet Gynecol. 2000;43(2):321-5.

14. Chang FM, Liang RI, Ko HC, Yao BL, Chang $\mathrm{CH}$, Yu CH. Three-dimensional ultrasound-assessed fetal thigh volumetry in predicting birth weight. Obstet Gynecol. 1997;90(3):331-9.

15. Liang RI, Chang FM, Yao BL, Chang $\mathrm{CH}$, Yu CH, Ko HC. Predicting birth weight by fetal upper-arm volume with use of three-dimensional ultrasonography. Am J Obstet Gynecol. 1997;177(3):632-8.

16. Song TB, Moore TR, Lee JI, Kim YH, Kim EK. Fetal weight prediction by thigh volume measurement with three-dimensional ultrasonography. Obstet Gynecol. 2000;96(2):157-61.

17. Hadlock FP, Harrist RB, Carpenter RJ, Deter RL, Park SK. Sonographic estimation of fetal weight. The value of femur length in addition to head and abdomen measurements. Radiology. 1984;150(2):535-40.

18. Jeanty P, Cousaert E, Cantraine F, Hobbins JC, Tack B, Struyven J. A longitudinal study of fetal limb growth. Am J Perinatol. 1984;1(2):136-44.

19. Shepard M, Filly RA. A standardized plane for biparietal diameter measurement. J Ultrasound Med. 1982;1(4):145-50.

20. Phelan JP, Smith CV, Broussard P, Small M. Amniotic fluid volume assessment with the four-quadrant technique at 36-42 weeks gestation. J Reprod Med. 1987;32(7):540-2.

21. Grannum PA, Berkowitz RL, Hobbins JC. The ultrasonic changes in the maturing placenta and their relation to fetal pulmonic maturity. Am J Obstet Gynecol. 1979;133(8):915-22.

22. Fleiss J. The design and analysis of clinical experiments analysis. New York: Wiley; 1986.

23. Neter J, Kutner M, Nachtsheim C, Wasserman W. Applied linear statistical models. $4^{\text {th }}$ ed. Ilinois: Richard D Irwing; 1996.

24. Favre R, Nisand G, Bettahar K, Grange G, Nisand I. Measurement of limb circumference with three-dimensional ultrasound for fetal weight estimation. Ultrasound Obstet Gynecol. 1993;3(3):176-9.

25. Catalano PM, Tyzbir ED, Allen SR, McBean JH, McAuliffe TL. Evaluation of fetal growth by estimation of neonatal body composition. Obstet Gynecol. $1992 ; 79(1): 46-50$

26. Schild RL, Fimmers R, Hansmann M. Fetal weight estimation by threedimensional ultrasound. Ultrasound Obstet Gynecol. 2000;16(5):445-52.

27. Schild RL, Maringa M, Siemer J, Meurer B, Hart N, Goecke TW, et al. Weight estimation by three-dimensional ultrasound imaging in the small fetus. Ultrasound Obstet Gynecol, 2008;32(2):168-75.

28. Lee W, Comstock CH, Kirk JS, Smith RS, Monck JW, Deenadayalu R, et al. Birthweight prediction by three-dimensional ultrasonographic volumes of the fetal thigh and abdomen. J Ultrasound Med. 1997;16(12):799-805.

29. Warsof SL, Gohari P, Berkowitz RL, Hobbins JC. The estimation of fetal weight by computer-assisted analysis. Am J Obstet Gynecol. 1977;128(8):881-92.

30. Lee W, Deter RL, Ebersole JD, Huang R, Blanckaert K, Romero R. Birth weight prediction by three-dimensional ultrasonography: fractional limb volume. J Ultrasound Med. 2001;20(12):1283-92.

31. Pang BS, Kot BC, Ying M. Three-dimensional ultrasound volumetric measurements: is the largest number of image planes necessary for outlining the region-of-interest? Ultrasound Med Biol. 2006; 32(8):1193-202.

Artigo recebido: 27/6/09

Aceito para publicação: 11/11/09 